\title{
Exploration of alcohol use disorder-associated brain miRNA-mRNA regulatory networks
}

\author{
Yolpanhchana Lim (iD ${ }^{1,2}$, Jennifer E. Beane-Ebel (iD) ${ }^{3}$, Yoshiaki Tanaka ${ }^{4,8}$, Boting Ning ${ }^{3}$, Christopher R. Husted ${ }^{3}$, David C. Henderson ${ }^{1}$, \\ Yangfei Xiang ${ }^{4,9}$, In-Hyun Park ${ }^{4}$, Lindsay A. Farrer $\mathbb{D}^{5,6,7}$ and Huiping Zhang $\mathbb{D}^{1,7}$ ख
}

(c) The Author(s) 2021

Transcriptomic changes in specific brain regions can influence the risk of alcohol use disorder (AUD), but the underlying mechanism is not fully understood. We investigated AUD-associated miRNA-mRNA regulatory networks in multiple brain regions by analyzing transcriptomic changes in two sets of postmortem brain tissue samples and ethanol-exposed human embryonic stem cell (hESC)derived cortical interneurons. miRNA and mRNA transcriptomes were profiled in 192 tissue samples (Set 1) from eight brain regions (amygdala, caudate nucleus, cerebellum, hippocampus, nucleus accumbens, prefrontal cortex, putamen, and ventral tegmental area) of 12 AUD and 12 control European Australians. Nineteen differentially expressed miRNAs (fold-change $>2.0 \& P<0.05$ ) and 97 differentially expressed mRNAs (fold-change $>2.0 \& P<0.001$ ) were identified in one or multiple brain regions of AUD subjects. AUDassociated miRNA-mRNA regulatory networks in each brain region were constructed using differentially expressed and negatively correlated miRNA-mRNA pairs. AUD-relevant pathways (including CREB Signaling, IL-8 Signaling, and Axonal Guidance Signaling) were potentially regulated by AUD-associated brain miRNA-mRNA pairs. Moreover, miRNA and mRNA transcriptomes were mapped in additional 96 tissue samples (Set 2) from six of the above eight brain regions of eight AUD and eight control European Australians. Some of the AUD-associated miRNA-mRNA regulatory networks were confirmed. In addition, miRNA and mRNA transcriptomes were analyzed in hESC-derived cortical interneurons with or without ethanol exposure, and ethanol-influenced miRNA-mRNA regulatory networks were constructed. This study provided evidence that alcohol could induce concerted miRNA and mRNA expression changes in reward-related or alcohol-responsive brain regions. We concluded that altered brain miRNA-mRNA regulatory networks might contribute to AUD development.

Translational Psychiatry (2021)11:504; https://doi.org/10.1038/s41398-021-01635-w

\section{INTRODUCTION}

Alcohol use disorder (AUD) is characterized by uncontrolled alcohol drinking due to physical and psychological dependence on alcohol. According to the 2019 National Survey on Drug Use and Health (NSDUH), AUD affects 14.1 million (4.2\%) adult Americans (8.9 million men and 5.2 million women) [1]. Mounting evidence suggests that AUD is a complex genetic disorder, with an estimated heritability of about $50 \%$ [2]. Besides genetic variation, chronic alcohol consumption can lead to neuroadaptive phenomena, such as alcohol tolerance, dependence, and withdrawal [3]. The underlying molecular mechanisms of alcohol-induced neuroadaptations has not been fully explored, but it is believed that gene expression changes in specific brain regions are associated with AUD development.

Studies with animal-based models and human postmortem brains have demonstrated that alcohol exposure alters the expression of genes involved in diverse cellular functions. Using $\mathrm{C} 57 \mathrm{BL} / 6 \mathrm{~J}$ mice as models, altered expression of immediate early genes (c-fos, fosB, and zif268) was observed in the hippocampus (HIP), the nucleus accumbens (NAc), the basolateral amygdala (AMY), and the lateral hypothalamus due to alcohol exposure [4]. Human postmortem brain studies have examined AUD-associated coding gene (or $\mathrm{mRNA}$ ) expression changes in three brain regions [prefrontal cortex (PFC), NAc, and ventral tegmental area (VTA)] comprising the core reward circuitry. Differentially expressed coding genes identified in postmortem PFC of AUD subjects are potentially involved in transcription [5], aldehyde detoxification [6], nicotine response and opioid signaling [7], oxidative stress $[5,8]$, mitochondrial function $[5,6]$, myelination [9-12], calcium signaling [11], protein trafficking [10], fatty acid metabolism [6], cell cycling [13], cell adhesion [12], and neuronal apoptosis $[5,11,12]$. Differentially expressed coding genes identified in postmortem NAC of AUD subjects may participate in synaptic transmission [5, 8], vesicle formation and cell architecture [5], transcription and lipid metabolism [14], and oxidative phosphorylation, mitochondrial dysfunction and cytokine signaling [15].

\footnotetext{
${ }^{1}$ Department of Psychiatry, Boston University School of Medicine, Boston, MA, USA. ${ }^{2}$ The Bioinformatics Program, Boston University Graduate School of Arts and Sciences, Boston, MA, USA. ${ }^{3}$ Section of Computational Biomedicine, Department of Medicine, Boston University School of Medicine, Boston, MA, USA. ${ }^{4}$ Department of Genetics, Yale University

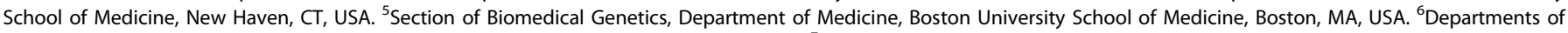
Neurology and Ophthalmology, Boston University School of Medicine, Boston, MA, USA. ${ }^{7}$ Departments of Biostatistics and Epidemiology, Boston University School of Public Health, Boston, MA, USA. ${ }^{8}$ Present address: Department of Medicine, Maisonneuve-Rosemont Hospital Research Center, University of Montreal, Montreal, QC H1T 2M4, Canada. ${ }^{9}$ Present address: School of Life Science and Technology, ShanghaiTech University, Shanghai 201210, China. ${ }^{\circledR e m a i l: ~ h u i p i n g z @ b u . e d u ~}$
}

Received: 14 May 2021 Revised: 3 September 2021 Accepted: 17 September 2021

Published online: 02 October 2021 
Only one study is known to have examined mRNA transcriptomic changes in postmortem VTA of AUD subjects, and the identified differentially expressed coding genes likely contribute to neurotransmission and signal transduction [8]. These findings suggest that altered expression of coding genes or mRNAs in rewardrelated brain regions may underlie alcohol-induced neuroadaptations.

AUD-associated mRNA expression changes can only partially explain the molecular mechanisms of AUD. Noncoding RNAs, particularly small noncoding microRNAs (miRNAs), have drawn much attention as they are potent and multifunctional regulators of many biological processes. miRNAs are a class of about 22 nucleotide-long small noncoding RNAs that act as regulators of gene expression at the post-transcriptional level. They bind to the $3^{\prime}$ untranslated region ( $3^{\prime}$ UTR) of their target mRNAs, resulting in either mRNA degradation (when their sequences are perfectly matched) or translational inhibition (when their sequences are imperfectly matched) [16]. The function of miRNAs implies an additional layer of gene expression regulation besides genetic variation. Accumulating evidence suggests that alcohol could induce miRNA expression changes, leading to altered cellular functions. Expression changes in miRNAs and their target mRNAs have been demonstrated as a consequence of exposure of alcohol to cultured cells $[17,18]$ as well as mouse [19] and rat [20-23] brains. miRNA transcriptomic changes have also been observed in postmortem PFC [13, 24] and NAc [15] of AUD subjects by microarray-based transcriptome analysis.

Given that AUD is a genetically heterogeneous disorder, it is commonly agreed that multiple genes (including both coding and noncoding genes) and the interactions among them contribute to the etiology of AUD. Studies have shown that a single miRNA can target hundreds of mRNA transcripts while a single mRNA transcript can be simultaneously regulated by distinct miRNAs [25]. The particular role of miRNAs in posttranscriptional regulation implies that miRNAs fine-tune the expression of numerous genes involved in a variety of cellular functions and thus coordinate multiple crosscommunicating pathways. Studies have demonstrated miRNA-mRNA regulatory networks were associated with psychiatric disorders such as schizophrenia [26]. However, no studies are known to have explored AUD-associated miRNA-mRNA regulatory networks.

Here, we report the first network analysis of AUD-associated brain miRNAs and mRNAs. Specifically, we examined AUDassociated miRNA and mRNA transcriptomic changes in multiple brain regions of AUD subjects. We also performed miRNA-mRNA pairing analysis and constructed AUD-associated and brain regionspecific miRNA-mRNA regulatory networks. To understand whether miRNA and mRNA expression changes in postmortem brains of AUD subject are due to alcohol consumption, we differentiated human embryonic stem cells (hESCs) into cortical interneurons and then used hESC-derived cortical interneurons as in vitro cellular models to examine ethanol-induced miRNA and mRNA transcriptomic changes. The convergence of multiple brain region transcriptome analysis and neuronal modeling could facilitate our understanding of the neuroadaptive mechanisms of AUD.

\section{MATERIALS AND METHODS}

\section{Human postmortem brain tissues}

Two sets of freshly-frozen autopsy brain tissue samples were obtained from the New South Wales Brain Tissue Resource Centre (NSWBTRC) in Australia. Set 1 included 480 [8 regions $\times(30$ cases +30 controls $)]$ postmortem tissue samples dissected from eight brain regions [amygdala (AMY), caudate nucleus (CN), cerebellum (CRB), hippocampus (HIP), nucleus accumbens (NAC), prefrontal cortex (PFC), putamen (PUT), and ventral tegmental area (VTA)] of 30 (21 male and 9 female) AUD and 30 (21 male and 9 female) control subjects (NSWBTRC approved project \#:
PID409). Set 2 included 360 [6 regions $\times(30$ cases +30 controls $)]$ postmortem tissue samples dissected from six brain regions (AMY, CN, CRB, HIP, PFC, and PUT) of 30 (20 male and 10 female) AUD and 30 (20 male and 10 female) control subjects (NSWBTRC approved project \#: PID191). All subjects were European Australians with no history of illicit drug abuse or major psychotic disorders (such as schizophrenia and bipolar disorder) according to the criteria in the Diagnostic and Statistical Manual of Mental Disorder 4th Edition (DSM-IV) [27]. Control subjects had no history of AUD.

Isolation and selection of brain tissue RNA samples for miRNA and mRNA transcriptome analysis

Total RNAs were isolated from 10 to $50 \mathrm{mg}$ of postmortem brain tissue samples using the miRNeasy Mini Kit (QIAGEN, Valencia, CA, USA). RNA integrity number (RIN) and concentration were measured using the Agilent 2100 Bioanalyser with the Agilent RNA 6000 Nano Kit (Agilent Technologies, Santa Clara, CA, USA). From the 480 Set 1 RNA samples, we selected 192 [from 8 brain regions of 12 AUD cases $(6$ males and 6 females) and 12 controls ( 6 males and 6 females)] with larger RINs (mean \pm SD: $6.6 \pm 1.3$ ) for miRNA and mRNA transcriptome analysis. From the 360 Set 2 RNA samples, we selected 96 (from 6 brain regions of 8 male AUD cases and 8 male controls) with larger RINs (mean \pm SD: $5.9 \pm 1.4$ ) for miRNA and mRNA transcriptome analysis. In both sets of selected RNA samples, cases and controls were matched by sex, age, RINs, and postmortem intervals (PMIs). Characteristics (including the amount of daily alcohol use, sex, age, PMIs, RINs, brain weight, brain $\mathrm{pH}$, cerebral hemispheres, smoking, and liver disease) of these two sets of RNA samples chosen for transcriptome analysis are summarized in Table S1. Except the amount of daily alcohol consumption, other demographic variables were not significantly different in their measurements (or counts) between cases and controls.

\section{RNA-seq analysis of miRNA and mRNA transcriptomic changes in eight brain regions of AUD subjects (192 Set 1 RNA samples)}

miRNA and mRNA expression profiles of the 192 selected Set 1 RNA samples were analyzed, respectively, by small RNA-seq and ribosome RNA (rRNA) depletion RNA-seq. Small RNA-seq was conducted as described in our previous study [28]. Briefly, small RNA-seq libraries were generated using the NEBNext Multiplex Small RNA Library Prep Set for Illumina (Set 1) (NEB, Ipswich, MA, USA) with $250 \mathrm{ng}$ of total RNAs. Purified cDNA libraries were pooled in equimolar ratios (12 libraries per pool) and multiplex sequenced at $1 \times 75 \mathrm{bp}$ on an Illumina HiSeq 2500 Sequencing System (Illumina, CA, USA). The Comprehensive Analysis Pipeline for miRNA Sequencing Data (CAP-miRseq) workflow [29] was used for raw reads (in fastq files) pre-processing, alignment, mature/precursor/novel miRNA qualification, and prediction. The mean total number of reads per sample was $16,591,602$, and the mean mapping rate (aligned reads/reads sent to Aligner) was $73.2 \%$. Principal component analysis (PCA) of miRNA transcriptome data of these 192 samples (from 8 brain regions) showed clustered CRB and VTA samples, but samples from six other brain regions could not be separated by brain regions using the miRNA expression data (Fig. S1a). The small RNA-seq fastq files and normalized read counts are available for downloading from the NCBI Gene Expression Omnibus (GEO) database (accession number: GSE181520).

Since most of the 192 selected postmortem brain RNA samples had RINs below 7 (Table S1), the KAPA RNA HyperPrep Kit with RiboErase (KAPA Biosystems, Wilmington, MA, USA) was used to deplete ribosomal RNAs (rRNAs) and construct RNA-seq libraries with $1 \mu \mathrm{g}$ of total RNAs as the starting material. Pooled libraries were loaded into individual lanes (80 pooled libraries/lane) of the NovaSeq S4 flow cell (Illumina, San Diego, CA, USA) by running the NovaSeq Xp workflow for $100 \mathrm{bp}$ paired-end sequencing on a NovaSeq $^{\text {TM }} 6000$ system (Illumina, San Diego, CA, USA). The bulk RNA-seq processing pipeline Pipeliner [30] was utilized to quantitate gene and isoform expression. The mean total number of reads per sample was $38,758,477$, and the mean mapping rate (aligned reads/reads sent to Aligner) was $84.6 \%$. PCA plotting of the mRNA-seq data showed similar sample clustering patterns as above using the miRNA-seq data (Fig. S1b). The rRNA depletion RNA-seq fastq files and normalized read counts are available for downloading from the NCBI GEO database (accession number: GSE181982).

Microarray analysis of miRNA and mRNA transcriptomic changes in six brain regions of AUD subjects (96 Set 2 RNA samples). For miRNA transcriptome analysis, the Affymetrix GeneChip ${ }^{\mathrm{TM}}$ miRNA4.0 array (Affymetrix, Santa 
Clara, CA, USA) was used following the manufacturer's instructions. This array was designed to detect all miRNAs in miRBase Release 20 [31]. It contains 30,424 probe sets for mature miRNAs of 203 species including 2578 human mature miRNA probe sets, 2025 human pre-miRNA probe sets, and 1996 human snoRNA and scaRNA probe sets. Briefly, over $100 \mathrm{ng}$ of total RNAs were labeled with the FlashTag ${ }^{\text {TM }}$ Biotin HSR Labeling Kit (ThermoFisher Scientific, Waltham, MA, USA) and hybridized to the array. The samples are washed, stained, and scanned using the Affymetrix Command Console and its associated robotics. Probe cell intensity files (or CEL files) for small noncoding RNAs (including miRNAs) were generated using the Affymetrix ${ }^{\circledast}$ GeneChip $^{T M}$ Command Console (AGCC) software. Small noncoding RNA CEL files were processed using the Affymetrix Expression Console (EC) software (v1.4.1) with the "MicroRNA Arrays - RMA (robust multi-array average) + DABG (detection above Background)Human only" workflow as the default analysis for background adjustment and signal normalization as well as $\log _{2}$ transformation to create probelevel summarization files (or CHP files). Quality control (QC) analysis of the CHP files was performed within the EC software, and the quality of the miRNA expression array data was visualized using box plots (Fig. S2a). The CHP files for case and control samples were further analyzed by statistical programs to identify differentially expressed miRNAs and other small noncoding RNAs. The Affymetrix miRNA expression data has been deposited in the NCBI GEO database (accession number: GSE180700).

For mRNA transcriptome analysis, the Affymetrix Clariom ${ }^{\mathrm{TM}} \mathrm{D}$ human array (Affymetrix, Santa Clara, CA, USA) was used following the manufacturer's instructions. This array allows interrogating more than 540,000 transcripts (including coding and long non-coding genes, exons, and alternative splicing events as well as rare transcripts) using over 6.7 million probes. About $500 \mathrm{ng}$ of total RNA was used in the Affymetrix Clariom D human array assay. Probe cell intensity files (or CEL files) for transcripts were generated using the AGCC software. They were then analyzed using the Affymetrix EC software (v1.4.1) with the "Gene Level RMA-Sketch (robust multi-array average with sketch quantile normalization)" workflow as the default analysis for background adjustment and signal normalization as well as $\log _{2}$ transformation to create probe-level summarization files (or CHP files). Quality control (QC) analysis of the CHP files was performed within the EC software, and the quality of the mRNA expression array data was visualized using box plots (Fig. S2b). The CHP files for case and control samples were further analyzed by statistical programs to identify differentially expressed mRNAs. The Affymetrix mRNA expression data has been deposited in the NCBI GEO database (accession number: GSE180722).

\section{Differentiation of hESCs into cortical interneurons and analysis of ethanol-induced miRNA and mRNA transcriptomic changes by RNA-seq}

hESC-derived cortical interneurons were used as cellular models for analyzing ethanol-induced miRNA and mRNA transcriptomic changes. $\mathrm{H} 1$ hESCs (WiCell Research Institute, Madison, USA) were differentiated into cortical interneurons as previously described [32]. Briefly, H1 hESCs were dissociated with accutase (STEMCELL Technologies, Vancouver, Canada) and cultured in mTeSR1 (STEMCELL Technologies, Vancouver, Canada) on Matrigel (Corning Life Science, Tewksbury, USA) coated plate until 95\% confluence. For neural induction (from Day 1 to Day 10), hESCs were cultured in the neural induction medium containing three inhibitors including $100 \mathrm{nM}$ of LDN-193189 (Stemgent, Cambridge, MA, USA), $10 \mu \mathrm{M}$ of SB-431542 (Tocris Bioscience, Bristol, UK), and $2 \mu \mathrm{M}$ of XAV-939 (Stemgent, Cambridge, MA, USA), and the neural induction medium was changed daily. For ventral patterning (Day 11-Day 18), the cells were cultured in neural induction media containing $100 \mathrm{ng} / \mathrm{ml}$ of SHH (R\&D Systems, Minneapolis, MN, USA) and $1 \mu \mathrm{M}$ of purmorphamine (Stemgent, Cambridge, MA, USA), and the medium was changed every other day. For final neuronal differentiation and maturation (Day 19 and after), the cells were cultured in the neuronal maturation medium supplemented with $20 \mathrm{ng} / \mathrm{ml}$ of BDNF (R\&D Systems, Minneapolis, MN, USA), $200 \mu \mathrm{M}$ of ascorbic acid (Sigma-Aldrich, St. Louis, MO, USA), and $200 \mu \mathrm{M}$ of CAMP (Sigma-Aldrich, St. Louis, MO, USA), and the medium was changed every 4 days. After 6 weeks of maturation (totally 62 days in vitro differentiation), the $\mathrm{H} 1 \mathrm{hESC}$-derived cortical interneurons were characterized by immunostaining (Fig. S3) to confirm the expression of neuronal biomarkers as described in our previous study [33].

hESC-derived cortical interneurons were then cultured in the neuronal maturation media containing ethanol at a concentration of around 50-100 mM (equivalent to blood alcohol levels of heavy or intoxicated drinkers) for 7 days. The ethanol-containing neuronal maturation medium was changed every other day. After additional 24-h culture without ethanol exposure, the cells were collected for total RNA isolation. Cell treatment experiments (exposed or unexposed to ethanol for 7 days) were performed in duplicate. Extra wells of cells treated with or without ethanol were fixed with $4 \%$ paraformaldehyde for cell morphology assay. Ethanolexposed cells did not show apparent morphological changes (Fig. S4).

miRNA transcriptomes of hESC-derived neurons (exposed or unexposed to ethanol) were profiled by small RNA-seq and the raw data obtained from small RNA-seq was processed by CAP-miRseq [29], as described above. The mean total number of reads per sample was $29,145,212$, and the mean mapping rate (aligned reads/reads sent to Aligner) was $85.0 \%$. The quality of the miRNA-seq data was visualized using box plots (Fig. S5a). The miRNA-seq data has been deposited in the NCBI GEO database (accession number: GSE181050).

mRNA-seq was applied to profile the mRNA transcriptome of hESC-derived cortical interneurons since high-quality total RNA samples (RINs $>7$ ) were extracted from cultured cells. The lllumina ${ }^{\circledR}$ TruSeq $^{\circledR}$ Stranded mRNA Library Prep Kit (lllumina, San Diego, CA, USA) was used to construct mRNA-seq libraries. Pooled cDNA libraries (up to 8 ) were sequenced $(2 \times 100 \mathrm{bp})$ on the HiSeq 2000 system (Illumina, San Diego, CA, USA). The mRNA-seq raw data was processed by Pipeliner [30], as described above. The mean total number of reads per sample was 25,524,900 and the mean mapping rate (aligned reads/reads sent to Aligner) was $75.1 \%$. The quality of the mRNA-seq data was visualized using box plots (Fig. S5b). The mRNA-seq data has been deposited in the NCBI GEO database (accession number: GSE181049).

\section{Statistical analyses}

Differential expression analysis was performed to identify differentially expressed miRNAs and mRNAs in each brain region of AUD subjects (given that gene expression is tissue-specific) and ethanol-exposed hESC-derived cortical interneurons. For RNA-seq data from Set 1 brain tissue samples, the voom method [34], which is a function of the limma package [35], was used to estimate the mean-variance relationship of the log-counts and generate a precision weight for each gene. The RNA-seq read counts information from the voom analysis was then entered into the empirical Bayes analysis pipeline. The Imfit function in the limma package [35] was then used to fit a linear regression model using the weighted least square for each gene, and comparisons between case and control groups in $\log _{2}$ fold-changes $\left(\log _{2} \mathrm{FC}\right)$ were obtained as contrasts of the fitted linear model, with a number of confounding factors being considered as covariates. We did principal component analysis (PCA) to extract the first three PCs for both technical (batch, RIN, and PMI) and biological (sex, age, brain weight, brain $\mathrm{pH}$, left-right brain, smoking, and liver disease) confounding variables, and the obtained PC1, PC2, and PC3 were used as covariates in the model matrix design for differential expression analysis, as described in a recent article [36]. For microarray expression data from Set 2 brain tissue samples, the differential expression analysis was performed in the same way using the Imfit function in the limma package [35].

\section{Bioinformatics analysis}

The function of differentially expressed miRNAs was annotated using DIANA TOOLS - mirPath v.3 [37]. The Gene Ontology (GO) analysis of molecular functions (MF), biological processes (BP), and cellular components (CC) overrepresented in differentially expressed mRNAs was conducted using DAVID v6.8 [38]. In addition, AUD-associated miRNA-mRNA pairs and their associated canonical pathways in each brain region were analyzed using the miRNA Target Filter function in Ingenuity Pathway Analysis (IPA, Ingenuity Systems, http://www.ingenuity.com). First, the differential expression analysis results [including fold changes $(\mathrm{FC})$ and $P$ values] of differentially expressed miRNAs (absolute $F C \geq 1.3$ and $P<0.05$ ) identified in each brain region were uploaded as the input miRNA dataset for the IPA miRNA Target Filter function, and miRNA-mRNA pairs were then revealed using predicted miRNA-mRNA interactions from TargetScan [39], TarBase [40], and miRecords [41] as well as microRNA-related findings from peer-reviewed literature. Second, the differential expression analysis results (including FC and $P$ values) of differentially expressed mRNAs (absolute $\mathrm{FC} \geq 1.3$ and $P<0.05$ ) identified in the same brain region were added, and the Expression Pairing function of the IPA miRNA Target Filter was applied to obtain miRNA-mRNA pairs in which their expression levels were negatively-correlated (i.e., upregulated miRNA-downregulated mRNA pairs or downregulated miRNA-upregulated mRNA pairs). Third, the obtained miRNA-mRNA pairs were used to construct miRNA-mRNA interaction networks. Finally, AUD-related canonical pathways 

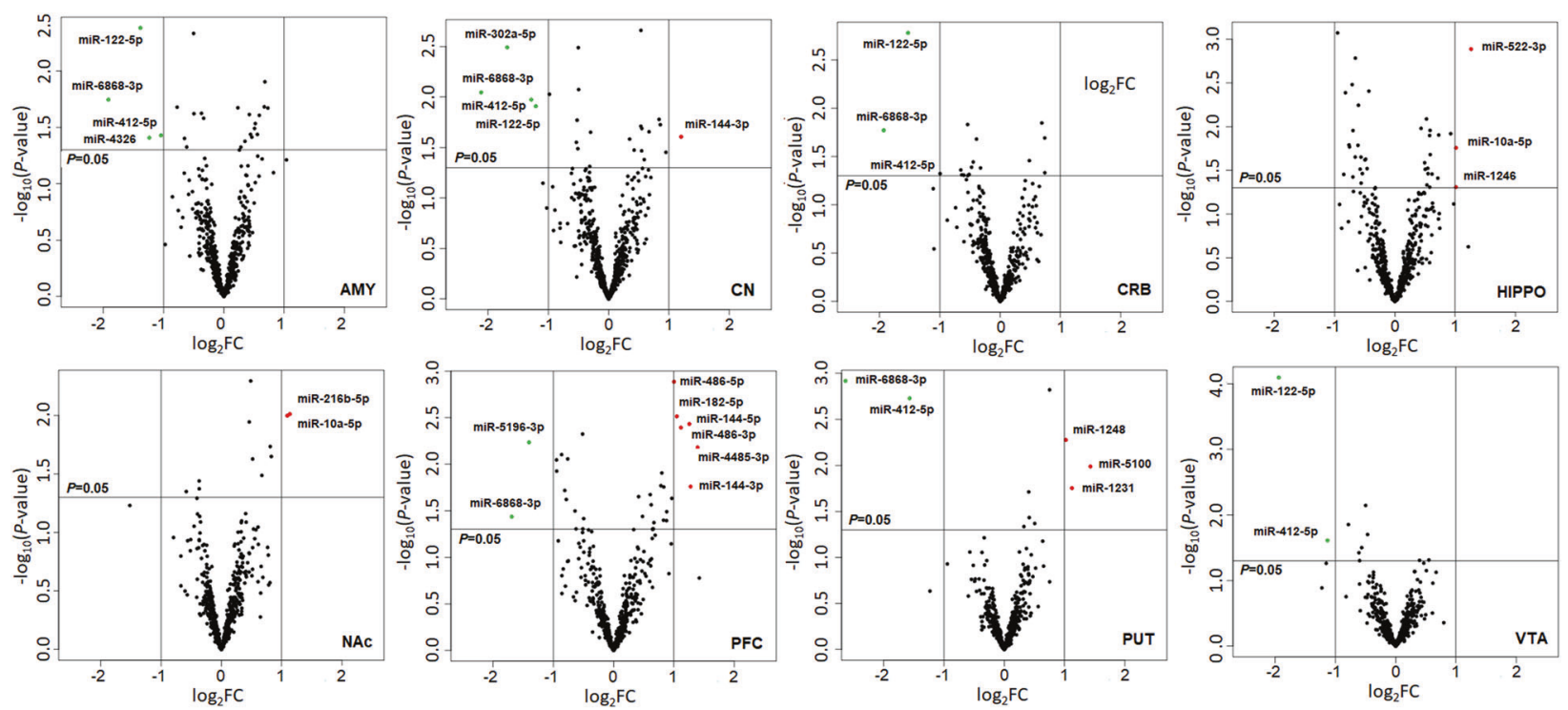

Fig. 1 Volcano plots displaying differentially expressed miRNAs in eight regions of postmortem brains of subjects with alcohol use disorder (AUD) (the Set 1 sample). The vertical axis ( $y$-axis) corresponds to the negative $\log _{10}$ of the $P$-value, and the horizontal axis ( $\mathrm{x}$-axis) displays the $\log _{2}$ of fold changes (FC). The red dots represent upregulated miRNAs ( $\left.\log _{2} F C>1.0 \& P<0.05\right)$ and the green dots represent downregulated miRNAs $\left(\log _{2} \mathrm{FC}<-1.0 \& P<0.05\right)$. The horizontal line shows the $P$-value cutoff $(P=0.05)$ with points above the line having the $P$-value $<0.05$ and points below the line having the $P$-value $>0.05$. The two vertical lines indicate 2 -fold changes. AMY amygdala, CN caudate nucleus, CRB cerebellum, HIPPO hippocampus, NAc nucleus accumbens, PFC prefrontal cortex, PUT putamen, VTA ventral tegmental area.

were added to miRNA-mRNA networks to display miRNA-mRNA-pathway relationships.

\section{RESULTS \\ Differentially expressed miRNAs in multiple brain regions of AUD subjects and ethanol-exposed hESC-derived cortical interneurons}

By small RNA-seq analysis of the 192 selected Set 1 samples (from 8 brain regions), we identified 19 differentially expressed mature miRNAs (absolute $\mathrm{FC}>2.0 \& P<0.05$ ) in one or more brain regions (4 in AMY, 5 in CN, 3 in CRB, 3 in HIP, 2 in NAC, 8 in PFC, 5 in PUT, and 2 in VTA) of AUD subjects (Fig. 1 and Table S2). Two miRNAs were upregulated $(>2$-fold increase $\& P<0.05)$ in multiple brain regions (miR-10a-5p: HIP and NAc; miR-144-3p: CN and PFC) of AUD subjects, while three other miRNAs were downregulated ( $>2$ fold decrease $\& P<0.05$ ) in multiple brain regions (miR-122-5p: AMY, CN, CRB, and VTA; miR-412-5p: AMY, CN, CRB, PUT, and VTA; and miR-6868-3p: AMY, CN, CRB, PFC, and PUT) of AUD subjects.

By Affymetrix miRNA 4.0 microarray analysis of the 96 selected Set 2 samples (from 6 brain regions), we identified 52 differentially expressed (absolute $F C>2.0 \& P<0.05$ ) miRNAs in one or more brain regions (1 in AMY, 1 in CN, 3 in CRB, 4 in HIP, 44 in PFC, and 4 in PUT) of AUD subjects (Fig. S6 and Table S3). Same as above, miR-412-5p was downregulated ( $>2$-fold decrease \& $P<0.05$ ) in five of the six brain regions (AMY, CN, CRB, HIP, and PUT) of AUD subjects.

We also examined ethanol-induced miRNA transcriptomic changes in hESC-derived cortical interneurons (as a cellular model) by small RNA-seq. A 7-day ethanol exposure led to differential expression of six miRNAs (absolute FC $>2.0 \& P<0.05$ ) in hESC-derived cortical interneurons. Three miRNAs (miR-151b, miR-151a-5p, and miR-3135a) were upregulated ( $>2$-fold increase $\& P<0.05$ ), while three other miRNAs (miR-548bc, miR-3609, and miR-493-5p) were downregulated ( $>2$-fold decrease \& $P<0.05$ ) due to ethanol exposure (Fig. S7 and Table S4). The expression of miR-412-5p, which was downregulated in multiple brain regions of AUD subjects, was on a decreasing trend (1.3-fold decrease \& $P$ $=0.263$ ) in ethanol-exposed hESC-derived cortical interneurons.

Venn diagrams were made to display the number of differentially expressed miRNAs $(P<0.05)$ shared between eight brain regions of AUD subjects (Set 1 and Set 2) and ethanol-exposed hESC-derived cortical interneurons (Fig. S8). The in vitro cellular model study confirmed several AUD-associated brain miRNAs, including miR-98$3 p$ (in AMY and HIP), miR-508-5p (in AMY), miR-548ah-3p (in CRB), miR-548p (in CRB), miR-486-5p (in PFC), miR-486-3p (in PFC), miR139-3p (in PFC), and miR-151a-5p (in PFC).

\section{Differentially expressed mRNAs in multiple brain regions of AUD subjects and ethanol-exposed hESC-derived cortical interneurons}

By rRNA depletion RNA-seq analysis of the 192 selected Set 1 samples (from 8 brain regions), we identified 97 differentially expressed (absolute $\mathrm{FC}>2.0 \& P<0.001$ ) coding genes (or mRNAs) in one or more brain regions (5 in AMY, 4 in CN, 21 in CRB, 11 in HIP, 4 in NAC, 46 in PFC, 11 in PUT, and 6 in VTA) of AUD subjects (Fig. 2 and Table S5). Three coding genes were upregulated ( $>2$-fold increase $\& P<$ 0.001 ) in multiple brain regions (CHI3L1: AMY, PUT, and VTA; FSIP2: CN, NAC, PFC, and PUT; and MAFB: CRB and VTA) of AUD subjects, while two other coding genes were downregulated ( $>2$-fold decrease $\& P<0.001)$ in multiple brain regions $(C Y Y R 1: C N, C R B$, and HIP; EDN3: CN, HIP, NAc, and PUT) of AUD subjects.

By Affymetrix Clarion D Human microarray analysis of the 96 selected Set 2 samples (from 6 brain regions), we identified six differentially expressed (absolute $\mathrm{FC}>2.0 \& P<0.001$ ) coding genes (or mRNAs) in one or more brain regions (upregulated OCLN and downregulated GLS2 in AMY; downregulated SLC47A1, PROX1, MYO5B, and TNC in HIP; and upregulated OCLN in PUT) of AUD subjects (Fig. S9 and Table S6). Decreased expression of TNC was also observed in multiple brain regions of Set 1 AUD subjects (AMY: 2.53-fold decrease $\& P=0.016 ; C N: 1.48$-fold decrease \& $P=0.307 ;$ HIP: 1.38-fold decrease $\& P=0.406$; and PFC: 2.61 -fold decrease $\& P=0.016$ ).

We also examined ethanol-induced mRNA transcriptome changes in hESC-derived cortical interneurons by mRNA-seq. A 7-day ethanol 

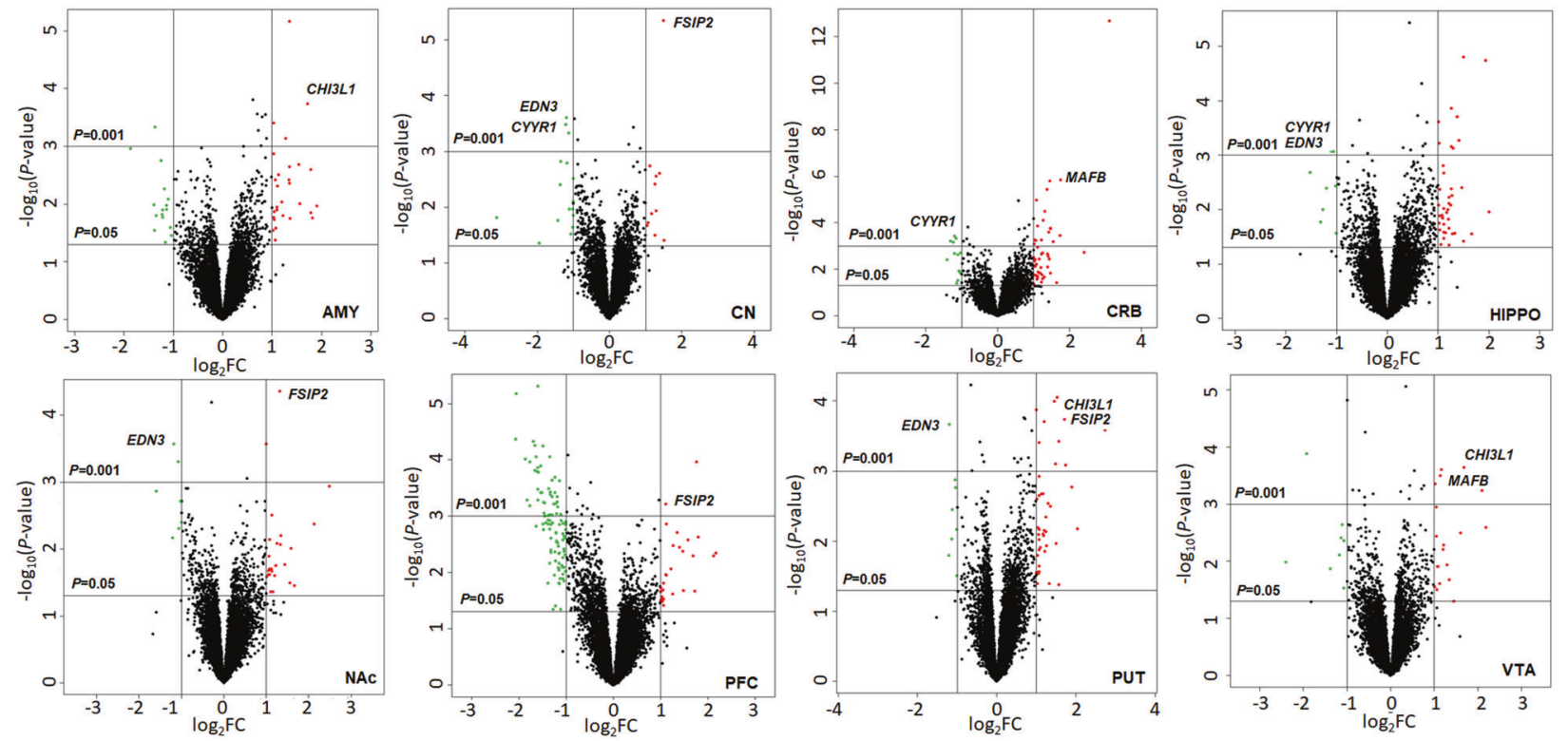

Fig. 2 Volcano plots displaying differentially expressed mRNAs in eight regions of postmortem brains of subjects with alcohol use disorder (AUD) (the Set 1 sample). The vertical axis ( $y$-axis) corresponds to the negative $\log _{10}$ of the $P$-value, and the horizontal axis ( $x$-axis) displays the $\log _{2}$ of fold changes (FC). The red dots represent upregulated mRNAs $\left(\log _{2} \mathrm{FC}>1.0 \& P<0.05\right)$ and the green dots represent downregulated mRNAs $\left(\log _{2} \mathrm{FC}<-1.0 \& P<0.05\right)$. The horizontal line shows the $P$-value cutoff $(P=0.05$ or 0.01$)$ with points above the line having the $P$-value $<0.05$ or 0.01 and points below the line having the $P$-value $>0.05$ or 0.01 . The two vertical lines indicate 2 -fold changes. AMY amygdala, CN caudate nucleus, CRB cerebellum, HIPPO hippocampus, NAc nucleus accumbens, PFC prefrontal cortex, PUT putamen, VTA ventral tegmental area.

exposure did not cause coding gene expression changes at the above significance level (absolute FC $>2.0 \& P<0.001$ ) (Fig. S10). When the significance level was set at $\mathrm{FC}>2.0 \& P<0.01,19$ coding genes showed differential expression, and all of them were downregulated after a 7-day ethanol exposure (Table S7).

Venn diagrams were used to show the number of differentially expressed mRNAs $(P<0.05)$ shared between eight brain regions of AUD subjects (Set 1 and Set 2) and ethanol-exposed hESC-derived cortical interneurons (Fig. S11). The in vitro cellular model study confirmed a number of AUD-associated mRNAs, including 15 mRNAs in the AMY, 15 mRNAs in the CN, five mRNAs in the CRB, five mRNAs in the HIP, seven mRNAs in the NAc, eight mRNAs in the PFC, 10 mRNAs in the PUT, and four mRNAs in the VTA. Among them, 13 AUD-associated coding genes identified in multiple brain regions were found differentially expressed in ethanol-exposed hESC-derived cortical interneurons (Fig. S11).

\section{Functional annotations of miRNAs and mRNAs differentially expressed in the brains of AUD subjects}

The function of the top 19 differentially expressed (absolute FC > $2.0 \& P<0.05$ ) miRNAs (Table S2) identified in one or more of the eight brain regions of AUD subjects (Set 1$)$ was annotated by DIANAmirPath. The top 14 KEGG pathways $(P<0.0001)$, including Morphine Addiction $\left(P=3.1 \times 10^{-8} ; 15\right.$ miRNAs), Cocaine Addiction $(P=1.5 \times$ $10^{-5} ; 15$ miRNAs), and Amphetamine Addiction $\left(P=1.0 \times 10^{-4} ; 15\right.$ miRNAs), were associated with mRNAs potentially targeted by these 19 miRNAs (Fig. 3). The function of the top 97 differentially expressed (absolute FC $>2.0 \& P<0.001$ ) mRNAs (Table S5) identified in one or more of the eight brain regions of AUD subjects (Set 1) was annotated by DAVID. The Gene Ontology (GO) enrichment analysis showed that several molecular functions (MF; such as Phosphatidate Phosphatase Activity), biological processes (BP; such as Central Nervous System Myelination), and cellular components (CC; such as Integral Component of Membrane) were enriched in these 97 differentially expressed mRNAs. GO terms (MF, BP, and CC) overrepresented $(P<0.05)$ for this gene set are displayed in Fig. S12.

\section{AUD-associated brain miRNA-mRNA regulatory networks}

Differentially expressed and negatively correlated miRNA-mRNA pairs were included in brain region-specific IPA network analysis. The differential expression analysis $P$ value was set at $<0.05$ and the absolute FC was set at $>1.3$ for both miRNAs and mRNAs. AUDassociated miRNA-mRNA-pathway networks for each of the eight brain regions (the Set 1 sample) are shown in Figs. 4 and 5, respectively. Within the AMY, 13 miRNAs (6 upregulated and 7 downregulated) and 13 paired mRNAs (10 upregulated and 3 downregulated) could regulate four pathways (CREB Signaling in Neurons, STAT3 Pathway, IL-8 Signaling, and Axonal Guidance Signaling) (Fig. 4a). Within the CN, 10 miRNAs (6 upregulated and 4 downregulated) and eight paired mRNAs ( 3 upregulated and 5 downregulated) could regulate four pathways (CREB Signaling in Neurons, Gap Junction Signaling, Axonal Guidance Signaling, and Neuroinflammatory Signaling) (Fig. 4b). Within the CRB, seven miRNAs ( 2 upregulated and 5 downregulated) and nine paired mRNAs (8 upregulated and 1 downregulated) could regulate three pathways (Synaptogenesis Signaling, CREB Signaling in Neurons, and Neuroinflammatory Signaling) (Fig. 4c). Within the HIP, 21 miRNAs (13 upregulated and 8 downregulated) and 15 paired mRNAs ( 9 upregulated and 6 downregulated) could regulate four pathways (G-Protein Coupled Receptor Signaling, CREB Signaling in Neurons, Synaptogenesis Signaling, and Axonal Guidance Signaling) (Fig. 4d). Moreover, within the NAc, two upregulated miRNAs and two paired downregulated mRNAs could regulate one pathway (i.e., CREB Signaling in Neurons) (Fig. 5a). Within the PFC, 19 miRNAs (14 upregulated and 5 downregulated) and 22 paired mRNAs (5 upregulated and 17 downregulated) could regulate four pathways (IL-8 Signaling, Axonal Guidance Signaling, CREB Signaling in Neurons, and G-Protein Coupled Receptor Signaling) (Fig. 5b). Within the PUT, four miRNAs (3 upregulated and 1 downregulated) and four paired mRNAs ( 2 upregulated and 2 downregulated) could regulate two pathways (Sirtuin Signaling and IL-8 Signaling) (Fig. 5c). Within the VTA, nine miRNAs (2 upregulated and 7 downregulated) and 10 mRNAs (9 upregulated and 1 downregulated) could regulate five 


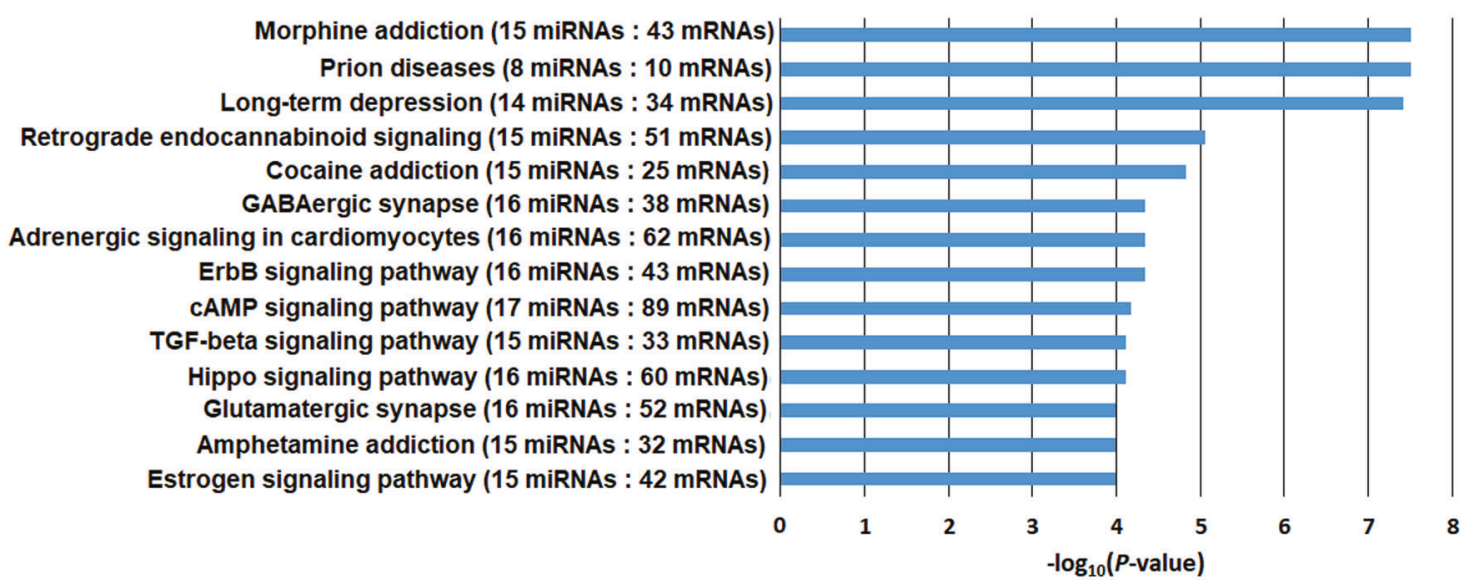

Fig. 3 DIANA-mirPath KEGG pathway enrichment analysis of mRNAs potentially targeted by 19 differentially expressed miRNAs (absolute FC $>2.0 \& P<0.05$ ) identified in one or multiple brain regions of subjects with alcohol use disorder (AUD) (the Set 1 sample). Numbers in parentheses: the number of differentially expressed miRNAs (absolute FC $>2.0 \& P<0.05$ ) and the number of predicted target mRNAs involved in specific pathways. KEGG pathways with enrichment $P$ values $<10^{-4}\left(\right.$ or $\left.-\log _{10} P>4.0\right)$ are listed.

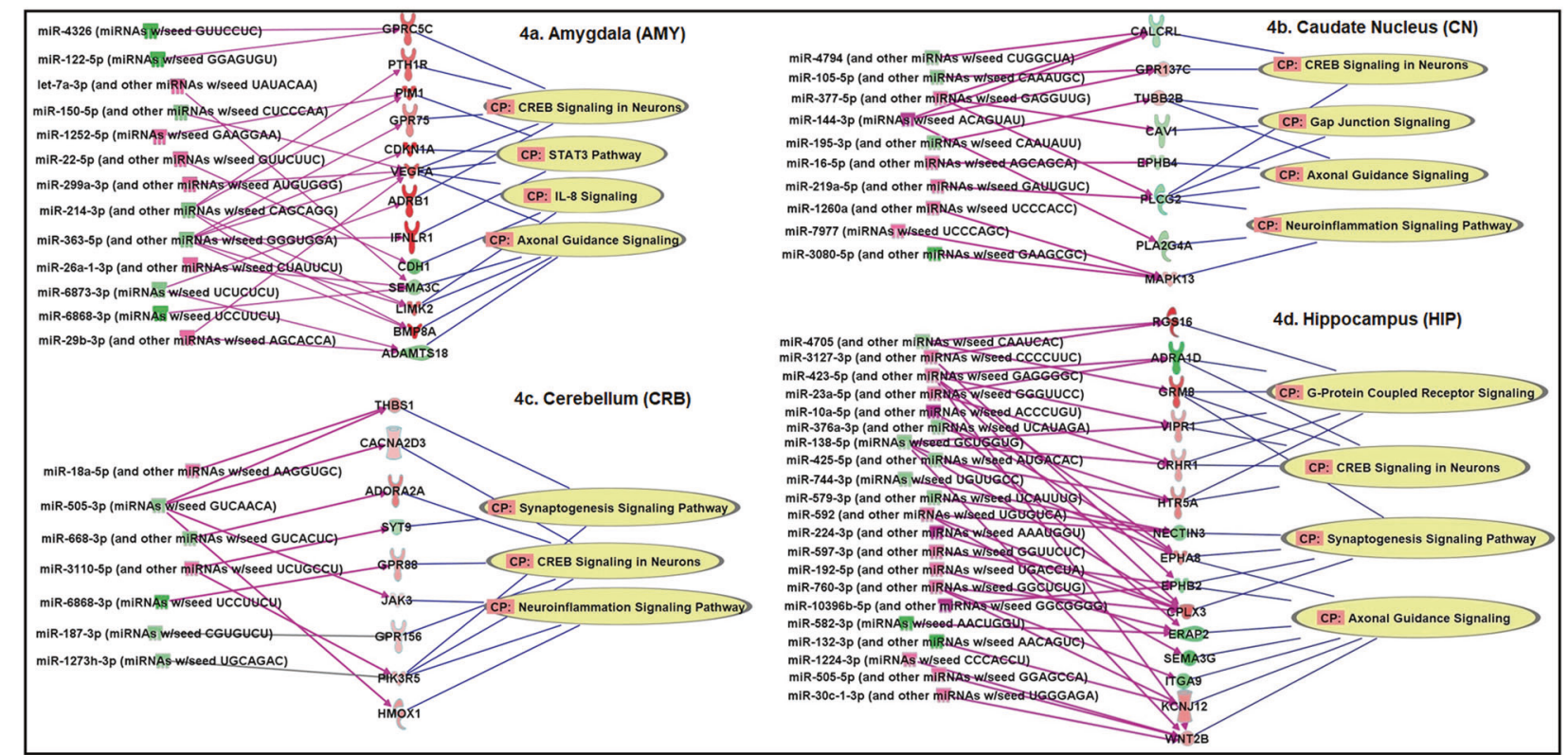

Fig. 4 Alcohol use disorder (AUD)-associated miRNA-mRNA regulatory networks in the amygdala (AMY), the caudate nucleus (CN), the cerebellum (CRB), and the hippocampus (HIP) of AUD subjects (the Set 1 sample). CP: Canonical pathways potentially regulated by differentially expressed [absolute fold-change $(F C)>1.3 \& P<0.05$ ] and negatively correlated miRNA-mRNA pairs identified in each brain region were defined using the Ingenuity Pathway Analysis (IPA) miRNA Target Filter function. miRNAs and mRNAs in red symbols: upregulated in AUD patients; miRNAs and mRNAs in green symbols: downregulated in AUD patients.

pathways (Opioid Signaling, CREB signaling in Neurons, IL-8 Signaling, NRF2-mediated Oxidative Stress Response, and Gap Junction Signaling) (Fig. $5 \mathrm{~d}$ ). Most of the above AUD-related pathways could also be regulated by differentially expressed (absolute FC $>1.3 \& P<0.05$ ) and negatively correlated miRNA-mRNA pairs identified in six of the above eight brain regions of AUD subjects (the Set 2 sample) (Figs. S13 and S14).

The analysis of miRNA-mRNA regulatory networks using differentially expressed (absolute $F C>1.3 \& P<0.05$ ) and negatively correlated miRNA-mRNA pairs identified in ethanol-exposed hESC-derived cortical interneurons indicated that four AUDrelevant pathways (Sirtuin Signaling, Opioid Signaling, NRF2mediated Oxidative Stress Response, and IL-1 Signaling) could be regulated by nine miRNAs ( 3 upregulated and 6 downregulated) and six paired mRNAs ( 1 upregulated and 5 downregulated) (Fig. S15). In total, 17 canonical pathways potentially regulated by differentially expressed and negatively correlated miRNA-mRNA pairs were identified in eight brain region of AUD subjects and ethanol-exposed hESC-derived cortical interneurons (Table S8). The top three pathways potentially regulated by AUD-associated and negatively correlated miRNA-mRNA pairs in multiple brain regions included CREB Signaling in Neurons, IL-8 Signaling, and Axonal Guidance Signaling. In addition, three AUD-associated miRNA-mRNA regulatory pathways (Opioid Signaling Pathway, NRF-mediated Oxidative Stress Response, and Sirtuin Signaling) were confirmed by the in vitro cellular model study (Table S8).

\section{DISCUSSION}

In this study, we observed miRNA and mRNA transcriptomic changes in multiple reward-related or alcohol-responsive brain regions of AUD subjects. We also discovered that brain region-specific 


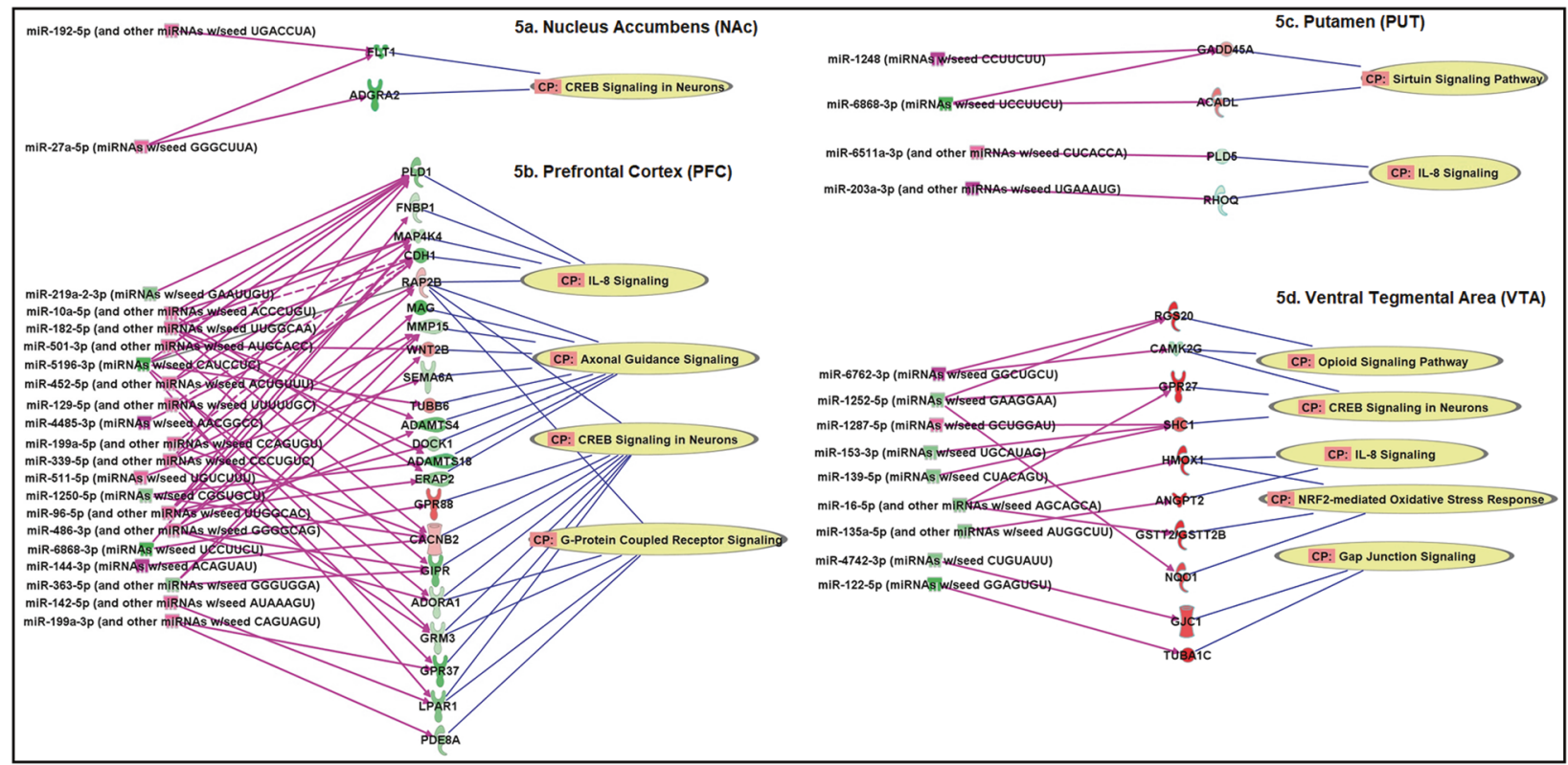

Fig. 5 Alcohol use disorder (AUD)-associated miRNA-mRNA regulatory networks in the nucleus accumbens (NAc), the prefrontal cortex (PFC), the putamen (PUT), and the ventral tegmental area (VTA) of AUD subjects (the Set 1 sample). CP: Canonical pathways potentially regulated by differentially expressed [absolute fold-change (FC) $>1.3 \& P<0.05$ ] and negatively correlated miRNA-mRNA pairs identified in each brain region were defined using the Ingenuity Pathway Analysis (IPA) miRNA Target Filter function. miRNAs and mRNAs in red symbols: upregulated in AUD patients; miRNAs and mRNAs in green symbols: downregulated in AUD patients.

miRNA-mRNA interactions potentially contribute to biological pathways important for AUD risk. Through the in vitro cellular model study, we validated that alcohol exposure could alter miRNA and mRNA expression profiles and miRNA-mRNA regulatory networks. To our knowledge, this is the first study that investigated the relationship of AUD and brain miRNA-mRNA regulatory networks.

First, transcriptomic changes identified by RNA-seq or microarray in multiple brain regions of AUD subjects suggest that several cortical and subcortical regions (or components of the reward circuit) are essential for the rewarding effect of alcohol. We observed miRNA and mRNA transcriptomic changes in eight reward-related or alcohol-responsive brain regions of AUD subjects. The reason that we chose these eight brain regions for this study is that they participate in brain functions such as motivation, memory, and pleasure as well as balance and locomotion [42-44]. Certainly, we cannot exclude the possibility that other brain regions also mediate the rewarding effect of ethanol or be involved in AUD-related pathways.

Second, brain regions with a larger number of AUD-associated miRNAs and mRNAs may be more responsive to alcohol stimulation or play a more important role in alcohol-induced neuroadaptations. As shown in Fig. 1, the PFC had the largest number of AUD-associated miRNAs, and there were more upregulated than downregulated miRNAs in the PFC. Correspondingly, more mRNAs were significantly downregulated in the PFC of AUD subjects than in other brain regions of AUD subjects (Fig. 2). Given the role of the PFC in higher cognitive functions, alcoholinduced expression changes of miRNAs and their target mRNAs in the PFC may lead to cognitive deficits and compromised working memory. Moreover, differentially expressed miRNAs and mRNAs were also observed in seven other brain regions of AUD subjects, and some AUD-associated miRNAs and mRNAs were shared among multiple brain regions of AUD subjects (Figs. 1 and 2). These findings provided insight into the coordinated role of multiple brain regions in AUD development and also suggested coordinated expression changes of miRNAs and mRNAs in the brains of AUD subjects.
Third, the findings that AUD-associated brain miRNAs potentially target genes involved in addiction-linked pathways suggest that these miRNAs play a critical role in AUD development. Through miRNA target gene prediction and pathway enrichment analyses by DIANA-mirPath, we found that the majority of the 19 differentially expressed miRNAs (Table S2) identified in one or more of the eight brain regions could target coding genes (or mRNAs) that participate in neurobiological processes of drug reward or addiction (Fig. 3). Among the top 14 pathways, four were related to drug addiction (Morphine Addition, Retrograde Endocannabinoid Signaling, Cocaine Addiction, and Amphetamine Addiction) and two were related to synaptic functions (GABAergic Synapse and Glutamatergic Synapse). Although alcohol and drugs of abuse (e.g., morphine and cocaine) possess diverse neuropharmacological potentials, their reinforcing effects are mediated by common pathways (such as dopaminergic and glutamatergic pathways) via the activation of the mesocorticolimbic system that are mainly comprised of the AMY, the NAc, the PFC, and the VTA [45]. That is to say, the above pathways for drug addiction or synaptic function can also mediate the rewarding effect of alcohol or are essential for neuroadaptive processes triggered by alcohol. Accordingly, AUD-associated miRNAs identified in the above eight brain regions are expected to regulate the expression of genes that are important for alcohol-induced neuroadaptations.

In addition, the present study provided evidence that brain miRNA-mRNA regulatory networks consisting of dysregulated and negatively correlated miRNA-mRNA pairs contribute to the risk of AUD. We identified at least 17 canonical pathways that were likely influenced by dysregulated and negatively correlated miRNA-mRNA pairs in the brains of AUD subjects (Table S8). The top three pathways potentially regulated by dysregulated and negatively correlated miRNA-mRNA pairs in multiple brain regions of AUD subjects included CREB Signaling in Neurons, IL-8 Signaling, and Axonal Guidance Signaling. The CREB Signaling was found to be a central amygdaloid signaling pathway involved in high anxiety-like and excessive alcohol drinking behaviors [46]. We found that the CREB Signaling pathway could be regulated by 
dysregulated and negatively correlated miRNA-miRNA pairs in seven of the eight brain regions (except PUT) of AUD subjects (Figs. 4 and 5). Regarding the relationship of the IL-8 Signaling pathway and AUD, there is emerging evidence that alcohol use can stimulate immune cells to secrete peripheral pro- and antiinflammatory cytokines (such as IL-8) $[47,48]$, thus supporting the role of the immune system in the pathophysiology of AUD. We observed that the IL-8 Signaling pathway could be regulated by dysregulated and negatively correlated miRNA-miRNA pairs in four (AMY, PFC, PUT, and VTA) of the eight brain regions of AUD subjects (Figs. 4 and 5). The Axon Guidance Signaling pathway can regulate axon guidance, synaptogenesis, and cell migration. Studies have shown that ethanol disrupted axon outgrowth by influencing the Axon Guidance Signaling pathway [49]. We noticed that the Axon Guidance Signaling pathway could be regulated by dysregulated and negatively correlated miRNA-miRNA pairs in four (AMY, CN, HIP, and PFC) of the eight brain regions of AUD subjects (Figs. 4 and 5). These three top pathways were validated in the Set 2 brain tissue sample by the network analysis of dysregulated and negatively correlated miRNA-mRNA pairs in six of the eight brain regions of AUD subjects (Figs. S13 and S14). Although these three top pathways were not found to be regulated by differentially expressed and negatively correlated miRNA-mRNA pairs identified in ethanol-exposed hESC-derived cortical interneurons, four other pathways [Sirtuin Signaling, Opioid Signaling, NRF2-mediated Oxidative Stress Response, and interleukin1 (IL-1) Signaling] were uncovered (Table S15). Except the IL-1 Signaling pathway, three other pathways were also identified by the analysis of Set 1 and Set 2 samples. Similar to the IL-8 Signaling pathway, the IL-1 Signaling pathway can also regulate immune response or inflammation caused by alcohol [50]. Therefore, multiple addiction-linked pathways influenced by AUD-associated miRNA-mRNA regulatory networks could contribute to the occurrence of AUD.

Some limitations of this study should be noted. First, bulk RNAseq cannot evaluate the functional relevance of miRNA-mRNA pairing at the cellular level. Since RNA samples for the transcriptome analysis were extracted from homogenized brain tissues, AUD-associated miRNA and mRNA expression changes may not occur in the same type of cells. To identify AUD-associated and cell type-specific miRNA-mRNA pairs, single-cell (or nucleus) RNA-seq can be applied to map miRNA and mRNA transcriptomes at the individual cell level. Second, the functional role of AUDassociated miRNA-mRNA networks in regulating neuronal function was not investigated. We only predicted by bioinformatics programs or based on published studies that a number of AUDrelated pathways were regulated by AUD-associated and negatively correlated miRNA-mRNA pairs. Animal model studies can be conducted to determine the influence of miRNA-mRNA interactions on neuronal function and addiction-related behaviors. Third, the transcriptome analysis of postmortem brain tissues cannot determine whether the dysregulation of brain miRNAs and mRNAs was due to pre-existing vulnerability factors (such as genetic variants and/or environmental insults) or long-term alcohol consumption. We intended to verify AUD-associated brain miRNA and mRNA changes using ethanol-exposed hESC-derived cortical interneurons as models. However, not many AUD-associated brain miRNAs and mRNAs were validated by the in vitro cellular model study. This is likely due to cell-type differences between brain tissues (containing many different types of neurons and glial cells) and hESC-derived neural cultures (mainly cortical interneurons and a small number of glial cells). To confirm whether AUD-associated brain miRNA and mRNA expression changes were indeed due to alcohol use and occur in a certain type of brain neuronal or glial cells, we could use controlled animal model studies and single-cell (or nucleus) RNA-seq.

We also found that many differentially expressed miRNAs and mRNAs identified in Set 1 brain tissue samples by RNA-seq were not confirmed in Set 2 brain tissue samples by microarray. The technical explanation is that RNA-seq can quantify a wider range of gene expression levels when compared to microarray. Although only about $78 \%$ of differentially expressed genes identified by microarray overlapped with those identified by RNA-seq even in the same set of RNA samples [51], a high correlation between these two platforms was observed in highly expressed genes that had less degradation or were more tolerant of degradation due to a larger number of transcripts [52]. The biological explanation for the inconsistent findings from Set 1 and Set 2 samples is that (1) brain tissues from the same brain region for RNA-seq and microarray were from different cohorts and they might not be dissected from the exactly same location and thus the brain tissue cell types could be slightly different, and (2) Set 1 samples consisted of both males and females while Set 1 samples were all males. Therefore, the biological differences between these two sets of samples may contribute more to the inconsistent results regardless of which platform was used. Similarly, the difference in cell types and proportions of different types of cells between hESC-derived neural cells and postmortem brain tissues is the most likely course why the in vitro cellular model study could not well confirm the findings from postmortem brain tissue studies. Moreover, we did not report AUD-associated and sexspecific transcriptomic changes when analyzing Set 1 samples because the subsample size (by sex) was too small to obtain unbiased results. In addition, we did not analyze other types of AUD-associated noncoding RNAs, such as long noncoding RNAs (IncRNAs). In the follow-up study, we will further analyze AUDassociated miRNA-mRNA-IncRNA regulatory networks.

In conclusion, the concerted expression changes of brain miRNAs and their target mRNAs as well as the interaction of them may govern alcohol-induced neuroplasticity, thus contributing to the development of AUD. To understand the mechanisms of the transition of alcohol use to abuse or dependence, the brain region-specific and single-cell spatialtemporal expression of brain miRNAs and their target mRNAs need to be investigated.

\section{REFERENCES}

1. SAMHSA. 2019 National Survey on Drug Use and Health (NSDUH). Substance Abuse and Mental Health Services Administration (SAMHSA); 2019.

2. Verhulst B, Neale MC, Kendler KS. The heritability of alcohol use disorders: a metaanalysis of twin and adoption studies. Psychol Med. 2015;45:1061-72.

3. Fadda F, Rossetti ZL. Chronic ethanol consumption: from neuroadaptation to neurodegeneration. Prog Neurobiol. 1998;56:385-431.

4. Ryabinin $A E$, Wang $Y M$, Freeman $P$, Risinger FO. Selective effects of alcohol drinking on restraint-induced expression of immediate early genes in mouse brain. Alcohol Clin Exp Res. 1999;23:1272-80.

5. Flatscher-Bader T, van der Brug M, Hwang JW, Gochee PA, Matsumoto I, Niwa S, et al. Alcohol-responsive genes in the frontal cortex and nucleus accumbens of human alcoholics. J Neurochem. 2005;93:359-70.

6. Zhang $\mathrm{H}$, Wang $\mathrm{F}, \mathrm{Xu} \mathrm{H}$, Liu Y, Liu J, Zhao H, et al. Differentially co-expressed genes in postmortem prefrontal cortex of individuals with alcohol use disorders: influence on alcohol metabolism-related pathways. Hum Genet. 2014;133:1383-94.

7. Kapoor M, Wang JC, Farris SP, Liu Y, McClintick J, Gupta I, et al. Analysis of whole genome-transcriptomic organization in brain to identify genes associated with alcoholism. Transl Psychiatry. 2019;9:89.

8. Flatscher-Bader T, van der Brug MP, Landis N, Hwang JW, Harrison E, Wilce PA. Comparative gene expression in brain regions of human alcoholics. Genes Brain Behav. 2006;5(Suppl 1):78-84.

9. Lewohl JM, Wang L, Miles MF, Zhang L, Dodd PR, Harris RA. Gene expression in human alcoholism: microarray analysis of frontal cortex. Alcohol Clin Exp Res. 2000;24:1873-82.

10. Mayfield RD, Lewohl JM, Dodd PR, Herlihy A, Liu J, Harris RA. Patterns of gene expression are altered in the frontal and motor cortices of human alcoholics. J Neurochem. 2002;81:802-13.

11. Iwamoto $K$, Bundo $M$, Yamamoto $M$, Ozawa $H$, Saito $T$, Kato T. Decreased expression of NEFH and PCP4/PEP19 in the prefrontal cortex of alcoholics. Neurosci Res. 2004;49:379-85. 
12. Liu J, Lewohl JM, Harris RA, lyer VR, Dodd PR, Randall PK, et al. Patterns of gene expression in the frontal cortex discriminate alcoholic from nonalcoholic individuals. Neuropsychopharmacology 2006;31:1574-82.

13. Lewohl JM, Nunez YO, Dodd PR, Tiwari GR, Harris RA, Mayfield RD. Up-regulation of microRNAs in brain of human alcoholics. Alcohol Clin Exp Res. 2011;35:1928-37.

14. Flatscher-Bader T, Harrison E, Matsumoto I, Wilce PA. Genes associated with alcohol abuse and tobacco smoking in the human nucleus accumbens and ventral tegmental area. Alcohol Clin Exp Res. 2010;34:1291-302.

15. Mamdani M, Williamson V, McMichael GO, Blevins T, Aliev F, Adkins A, et al. Integrating mRNA and miRNA weighted gene co-expression networks with eQTLs in the nucleus accumbens of subjects with alcohol dependence. PLoS ONE. 2015;10:e0137671.

16. Bartel DP. MicroRNAs: target recognition and regulatory functions. Cell 2009;136:215-33.

17. Yadav S, Pandey A, Shukla A, Talwelkar SS, Kumar A, Pant AB, et al. miR-497 and miR-302b regulate ethanol-induced neuronal cell death through $B C L 2$ protein and cyclin D2. J Biol Chem. 2011;286:37347-57.

18. Guo Y, Chen Y, Carreon S, Qiang M. Chronic intermittent ethanol exposure and its removal induce a different miRNA expression pattern in primary cortical neuronal cultures. Alcohol Clin Exp Res. 2012;36:1058-66.

19. Wang LL, Zhang Z, Li Q, Yang R, Pei X, Xu Y, et al. Ethanol exposure induces differential microRNA and target gene expression and teratogenic effects which can be suppressed by folic acid supplementation. Hum Reprod. 2009;24:562-79

20. Pietrzykowski AZ, Friesen RM, Martin GE, Puig SI, Nowak CL, Wynne PM, et al Posttranscriptional regulation of BK channel splice variant stability by miR-9 underlies neuroadaptation to alcohol. Neuron 2008;59:274-87.

21. Li J, Li J, Liu X, Qin S, Guan Y, Liu Y, et al. MicroRNA expression profile and functional analysis reveal that miR-382 is a critical novel gene of alcohol addiction. EMBO Mol Med. 2013;5:1402-14.

22. Coleman LG Jr, Zou J, Crews FT. Microglial-derived miRNA let-7 and HMGB1 contribute to ethanol-induced neurotoxicity via TLR7. J Neuroinflammation. 2017;14:22.

23. Asimes A, Kim CK, Rao YS, Bartelt K, Pak TR. microRNA expression profiles in the ventral hippocampus during pubertal development and the impact of peripubertal binge alcohol exposure. Noncoding RNA. 2019;5:21.

24. Wang F, Gelernter J, Zhang $\mathrm{H}$. Differential expression of miR-130a in postmortem prefrontal cortex of subjects with alcohol use disorders. J Addict Res Ther. 2013;4:18179.

25. Lim LP, Lau NC, Garrett-Engele P, Grimson A, Schelter JM, Castle J, et al. Microarray analysis shows that some microRNAs downregulate large numbers of target mRNAs. Nature 2005;433:769-73.

26. Guo AY, Sun J, Jia P, Zhao Z. A novel microRNA and transcription factor mediated regulatory network in schizophrenia. BMC Syst Biol. 2010;4:10.

27. American Psychiatric Association. American Psychiatric Association: diagnostic and statistical manual of mental disorders, 4th ed. Washington, DC: American Psychiatric Association. 1994.

28. Rosato AJ, Chen X, Tanaka Y, Farrer LA, Kranzler HR, Nunez YZ, et al. Salivary microRNAs identified by small RNA sequencing and machine learning as potential biomarkers of alcohol dependence. Epigenomics 2019;11:739-49.

29. Sun Z, Evans J, Bhagwate A, Middha S, Bockol M, Yan H, et al. CAP-miRSeq: a comprehensive analysis pipeline for microRNA sequencing data. BMC Genomics. 2014;15:423.

30. Federico A, Karagiannis T, Karri K, Kishore D, Koga Y, Campbell JD, et al. Pipeliner: a nextflow-based framework for the definition of sequencing data processing pipelines. Front Genet. 2019;10:614.

31. Kozomara A, Griffiths-Jones S. miRBase: integrating microRNA annotation and deep-sequencing data. Nucleic Acids Res. 2011;39:D152-57.

32. Maroof AM, Keros S, Tyson JA, Ying SW, Ganat YM, Merkle FT, et al. Directed differentiation and functional maturation of cortical interneurons from human embryonic stem cells. Cell Stem Cell. 2013;12:559-72.

33. Xiang Y, Kim KY, Gelernter J, Park IH, Zhang H. Ethanol upregulates NMDA receptor subunit gene expression in human embryonic stem cell-derived cortical neurons. PLoS ONE. 2015;10:e0134907.

34. Law CW, Chen Y, Shi W, Smyth GK. voom: Precision weights unlock linear model analysis tools for RNA-seq read counts. Genome Biol. 2014;15:R29.

35. Ritchie ME, Phipson B, Wu D, Hu Y, Law CW, Shi W, et al. limma powers differential expression analyses for RNA-sequencing and microarray studies. Nucleic Acids Res. 2015;43:e47.

36. Drake J, McMichael GO, Vornholt ES, Cresswell K, Williamson V, Chatzinakos C, et al. Assessing the role of long noncoding RNA in nucleus accumbens in subjects with alcohol dependence. Alcohol Clin Exp Res. 2020;44:2468-80.
37. Vlachos IS, Zagganas K, Paraskevopoulou MD, Georgakilas G, Karagkouni D, Vergoulis $\mathrm{T}$, et al. DIANA-miRPath v3.0: deciphering microRNA function with experimental support. Nucleic Acids Res. 2015;43:W460-66.

38. Dennis G Jr, Sherman BT, Hosack DA, Yang J, Gao W, Lane HC, et al. DAVID: database for annotation, visualization, and integrated discovery. Genome Biol. 2003;4:P3.

39. Agarwal V, Bell GW, Nam JW, Bartel DP. Predicting effective microRNA target sites in mammalian mRNAs. Elife 2015;4:e05005.

40. Vlachos IS, Paraskevopoulou MD, Karagkouni D, Georgakilas G, Vergoulis T, Kanellos I, et al. DIANA-TarBase v7.0: indexing more than half a million experimentally supported miRNA:mRNA interactions. Nucleic Acids Res. 2015;43:D153-59.

41. Xiao F, Zuo Z, Cai G, Kang S, Gao X, Li T. miRecords: an integrated resource for microRNA-target interactions. Nucleic Acids Res. 2009;37:D105-10.

42. Pascual M, Boix J, Felipo V, Guerri C. Repeated alcohol administration during adolescence causes changes in the mesolimbic dopaminergic and glutamatergic systems and promotes alcohol intake in the adult rat. J Neurochem. 2009;108:920-31.

43. Vertes RP. Interactions among the medial prefrontal cortex, hippocampus and midline thalamus in emotional and cognitive processing in the rat. Neuroscience 2006;142:1-20.

44. Zahr NM, Pitel AL, Chanraud S, Sullivan EV. Contributions of studies on alcohol use disorders to understanding cerebellar function. Neuropsychol Rev. 2010;20:280-89.

45. Feltenstein MW, See RE. The neurocircuitry of addiction: an overview. Br J Pharm. 2008;154:261-74.

46. Wand G. The anxious amygdala: CREB signaling and predisposition to anxiety and alcoholism. J Clin Investig. 2005;115:2697-99.

47. Loftis JM, Valerio J, Taylor J, Huang E, Hudson R, Taylor-Young P, et al. S100B and inflammatory cytokine levels in blood as potential markers of blood-brain barrier damage and psychiatric impairment in comorbid hepatitis $C$ viral infection and alcohol use disorder. Alcohol Clin Exp Res. 2018. https://doi.org/10.1111/acer.13796.

48. Bailey KL, Wyatt TA, Katafiasz DM, Taylor KW, Heires AJ, Sisson JH, et al. Alcohol and cannabis use alter pulmonary innate immunity. Alcohol 2019;80:131-38.

49. Chen S, Charness ME. Ethanol disrupts axon outgrowth stimulated by netrin-1, GDNF, and L1 by blocking their convergent activation of Src family kinase signaling. J Neurochem. 2012;123:602-12.

50. Mandrekar P, Dolganiuc A, Bellerose G, Kodys K, Romics L, Nizamani R, et al. Acute alcohol inhibits the induction of nuclear regulatory factor kappa $B$ activation through CD14/toll-like receptor 4, interleukin-1, and tumor necrosis factor receptors: a common mechanism independent of inhibitory kappa B alpha degradation? Alcohol Clin Exp Res. 2002;26:1609-14.

51. Rao MS, Van Vleet TR, Ciurlionis R, Buck WR, Mittelstadt SW, Blomme E, et al. Comparison of RNA-Seq and microarray gene expression platforms for the toxicogenomic evaluation of liver from short-term rat toxicity studies. Front Genet. 2018;9:636.

52. Rai MF, Tycksen ED, Sandell LJ, Brophy RH. Advantages of RNA-seq compared to RNA microarrays for transcriptome profiling of anterior cruciate ligament tears. J Orthop Res. 2018:36:484-97.

\section{ACKNOWLEDGEMENTS}

This work was supported by the National Institute on Alcohol Abuse and Alcoholism (NIAAA) grants R01AA025080 (HZ) and R21AA023068 (HZ). The authors are grateful to the New South Wales Brain Tissue Resource Centre (NSWBTRC) at The University of Sydney, Australia for providing alcoholic and control brain tissues for this study. The NSWBTRC is supported by the University of Sydney, the National Health and Medical Research Council of Australia, and the National Institute on Alcohol Abuse and Alcoholism (NIAAA). We also thank the deceased subjects' next of kin for providing informed written consent for the studies. In addition, the authors wish to thank Christopher Castaldi and Bryan Pasqualucci at the Yale Center for Genome Analysis (YCGA) as well as Mei Zhong and Ee-Chun Cheng at the Yale Stem Cell Center (YSCC) for helping with the RNA sequencing experiments.

\section{AUTHOR CONTRIBUTIONS}

$\mathrm{HZ}$ designed the experiments. $\mathrm{HZ}$ and $\mathrm{YX}$ conducted the experiments. $\mathrm{YL}, \mathrm{JEB}-\mathrm{E}, \mathrm{YT}$ $\mathrm{BN}, \mathrm{CRH}$, and $\mathrm{HZ}$ analyzed the data. $\mathrm{HZ}$ and $\mathrm{YL}$ wrote the manuscript. $\mathrm{DCH}, \mathrm{I-HP}$, and LAF edited the manuscript.

\section{COMPETING INTERESTS}

The authors declare no competing interests. 


\section{ADDITIONAL INFORMATION}

Supplementary information The online version contains supplementary material available at https://doi.org/10.1038/s41398-021-01635-w.

Correspondence and requests for materials should be addressed to Huiping Zhang.

Reprints and permission information is available at http://www.nature.com/ reprints

Publisher's note Springer Nature remains neutral with regard to jurisdictional claims in published maps and institutional affiliations.
Open Access This article is licensed under a Creative Commons Attribution 4.0 International License, which permits use, sharing, adaptation, distribution and reproduction in any medium or format, as long as you give appropriate credit to the original author(s) and the source, provide a link to the Creative Commons license, and indicate if changes were made. The images or other third party material in this article are included in the article's Creative Commons license, unless indicated otherwise in a credit line to the material. If material is not included in the article's Creative Commons license and your intended use is not permitted by statutory regulation or exceeds the permitted use, you will need to obtain permission directly from the copyright holder. To view a copy of this license, visit http://creativecommons.org/licenses/by/4.0/.

(c) The Author(s) 2021 